\title{
Interplay of Surface and Dirac Plasmons in Topological Insulators: The Case of $\mathrm{Bi}_{2} \mathrm{Se}_{3}$
}

\author{
A. Politano, ${ }^{1,{ }^{*}}$ V. M. Silkin, ${ }^{2,3,4}$ I. A. Nechaev, ${ }^{2,5,6}$ M. S. Vitiello, ${ }^{7}$ L. Viti, ${ }^{7}$ Z. S. Aliev, ${ }^{2,8,9}$ M. B. Babanly, ${ }^{8}$ G. Chiarello, ${ }^{1,10}$ \\ P. M. Echenique, ${ }^{2,3,11}$ and E. V. Chulkov ${ }^{2,3,5,11,6}$ \\ ${ }^{1}$ Department of Physics, University of Calabria, 87036 Rende (CS), Italy \\ ${ }^{2}$ Donostia International Physics Center (DIPC), Paseo de Manuel Lardizabal 4, 20018 San Sebastián/Donostia, Spain \\ ${ }^{3}$ Departamento de Física de Materiales, Universidad del País Vasco, Apartado 1072, 20080 San Sebastián/Donostia, Spain \\ ${ }^{4}$ IKERBASQUE, Basque Foundation for Science, 48011 Bilbao, Spain \\ ${ }^{5}$ Tomsk State University, 634050 Tomsk, Russian Federation \\ ${ }^{6}$ Saint Petersburg State University, 198504 Saint Petersburg, Russian Federation \\ ${ }^{7}$ NEST, Istituto Nanoscienze-CNR and Scuola Normale Superiore, Piazza San Silvestro 12, 56127 Pisa, Italy \\ ${ }^{8}$ Institute of Catalysis and Inorganic Chemistry, ANAS, AZ1143 Baku, Azerbaijian \\ ${ }^{9}$ Institute of Physics, ANAS, AZ1143 Baku, Azerbaijian \\ ${ }^{10}$ CNISM, Consorzio Nazionale Interuniversitario per le Scienze Fisiche della Materia, Via della Vasca Navale, 84, 00146 Roma, Italy \\ ${ }^{11}$ Centro de Física de Materiales CFM-Materials Physics Center MPC, Centro Mixto CSIC-UPV/EHU, \\ Paseo de Manuel Lardizabal 5, 20018 San Sebastián/Donostia, Spain
}

(Received 9 May 2015; revised manuscript received 19 August 2015; published 18 November 2015)

\begin{abstract}
We have investigated plasmonic excitations at the surface of $\mathrm{Bi}_{2} \mathrm{Se}_{3}(0001)$ via high-resolution electron energy loss spectroscopy. For low parallel momentum transfer $q_{\|}$, the loss spectrum shows a distinctive feature peaked at $104 \mathrm{meV}$. This mode varies weakly with $q_{\|}$. The behavior of its intensity as a function of primary energy and scattering angle indicates that it is a surface plasmon. At larger momenta $\left(q_{\|} \sim 0.04 \AA^{-1}\right)$, an additional peak, attributed to the Dirac plasmon, becomes clearly defined in the loss spectrum. Momentum-resolved loss spectra provide evidence of the mutual interaction between the surface plasmon and the Dirac plasmon of $\mathrm{Bi}_{2} \mathrm{Se}_{3}$. The proposed theoretical model accounting for the coexistence of three-dimensional doping electrons and two-dimensional Dirac fermions accurately represents the experimental observations. The results reveal novel routes for engineering plasmonic devices based on topological insulators.
\end{abstract}

DOI: 10.1103/PhysRevLett.115.216802

PACS numbers: 73.20.Mf, 79.20.Uv

In recent years, topological insulators (TIs) [1] (in particular $\mathrm{Bi}_{2} \mathrm{Se}_{3}$ ) have attracted considerable attention in the scientific community [2,3]. Topological surface states forming a Dirac cone [4-6] have promising capabilities in regard to engineering high-speed plasmonic and optoelectronic devices $[7,8]$.

The growth of TI samples belonging to the class of chalcogenides inherently involves the presence of vacancies. Thus, in most cases, the Fermi level is shifted from the bulk band gap and pinned by the bulk states occupied by doping electrons or holes. These bulk carriers give rise to their own three-dimensional electron gas (3DEG), which is well known to have plasmonic excitations characterized by the bulk plasmon energy $\omega_{p}$. In this matter, the frequency of the surface plasmon $\omega_{\mathrm{sp}}$ is related to the bulk plasmon energy $\omega_{p}$ (for a simple planar interface $\omega_{\mathrm{sp}}=\omega_{p} / \sqrt{1+\varepsilon_{b}}$, where $\varepsilon_{b}$ is the dielectric constant of the background medium of the 3DEG) [9]. In turn, the value of $\omega_{p}$ is determined by the bulk concentration of charge carriers [10]. From the reported values of the carrier concentration, the respective values of $\omega_{p}$, and the dielectric screening by the surrounding medium [11,12], one could expect that $\omega_{\mathrm{sp}}$ is in the $\sim 100 \mathrm{meV}$ energy range and it should coexist with the collective excitation of the two-dimensional electron gas (2DEG) arising from electrons of topological surface states (the so-called Dirac plasmon) $[13,14]$. As a result, the intriguing interplay of the surfaceplasmon and Dirac-plasmon modes as functions of in-plane momentum $q_{\|}$arises.

Recently, optical techniques have been used $[12,15,16]$ to investigate plasmon modes of thin films and microribbons of $\mathrm{Bi}_{2} \mathrm{Se}_{3}$. The Dirac plasmon has been observed in $\mathrm{Bi}_{2} \mathrm{Se}_{3}$ microribbons [15], while the bulk plasmon has been reported in thin films $[11,17,18]$. However, optical techniques are not suited to the investigation of the momentum dependence of plasmonic excitations and, thus, the nature and the dispersion of low-energy collective excitations in TIs in a finite momentum range is hitherto unexplored.

Here, we examine the surface collective electronic excitations in $\mathrm{Bi}_{2} \mathrm{Se}_{3}$ by means of HREELS, which allows probing of an extended momentum range. Our HREELS measurements provide direct evidence for the coexistence of two low-energy collective modes: the surface plasmon, whose origin is due to a nonzero density of $3 \mathrm{D}$ doping electrons, and the Dirac plasmon of the 2DEG formed by electrons residing in a topological surface-state band. The surface plasmon, whose surface nature is revealed by its cross section dependence on the scattering geometry and on the primary electron beam energy, has the energy of 
$\sim 100 \mathrm{meV}$ in the long-wavelength limit. In the neighborhood of $q_{\|}=0.04 \AA^{-1}$, the Dirac plasmon appears in the loss spectrum and its intensity increases for higher values of $q_{\|}$, thus becoming the dominant feature.

In the present work, a single crystalline ingot of $\mathrm{Bi}_{2} \mathrm{Se}_{3}$ was grown from melt by the vertical Bridgman-Stockbarger method. The polycrystalline sample which was synthesized from starting elements of a high purity grade (not less than $99.999 \%$ ) was placed in the conical-bottom quartz ampoule, which was sealed in a vacuum of less than $10^{-5} \mathrm{~Pa}$. Before the growth process, the ampoule was held in the "hot" zone $(1050 \mathrm{~K})$ of the two-zone tube furnace for $12 \mathrm{~h}$ to completely melt the composition. Then, the charged ampoule moved from the hot zone to the "cold" zone with the required rate of $1.0 \mathrm{~mm} / \mathrm{h}$. The temperature of the cold zone was about $900 \mathrm{~K}$. The grown crystals consisted of one large single crystalline block. Fresh surfaces were readily made available for HREELS measurements by cleaving the crystal along its natural cleavage plane. Such single crystals are also chemically inert $[19,20]$.

The cleaved $\mathrm{Bi}_{2} \mathrm{Se}_{3}(0001)$ samples were characterized in situ via a combination of angle-resolved photoemission spectroscopy (ARPES), scanning tunneling microscopy, AES, XPS, and LEED, and ex situ by XRD, AFM, and SEM to examine the crystalline quality, cleanliness, and the flatness of the cleaved surface. Transport experiments are reported in the Supplemental Material [20]. A well-defined Dirac cone was revealed via ARPES measurements (not shown). The $\mathrm{Bi}_{2} \mathrm{Se}_{3}(0001)$ surface exhibited an excellent $(1 \times 1)$ hexagonal LEED pattern characterized by sharp spots against a very low background. AES and vibrational spectra indicate the absence of any contamination at the surface. HREELS experiments were carried out in an ultrahigh vacuum chamber operating at a base pressure of $5 \times 10^{-9} \mathrm{~Pa}$, equipped with standard facilities for surface characterizations. HREELS spectra were acquired by using an electron energy loss spectrometer (Delta 0.5, SPECS). The energy resolution of the spectrometer was degraded to $5 \mathrm{meV}$ so as to increase the signal-to-noise ratio of loss peaks. The dispersion of the loss peaks, i.e., $E_{\text {loss }}\left(q_{\|}\right)$, was measured by moving the analyzer while keeping the sample and the monochromator in a fixed position.

To measure the dispersion relation, values for the parameters $E_{p}$, impinging energy, and $\alpha_{i}$, the incident angle, were chosen so as to obtain the highest signal-tonoise ratio. The range of primary beam energies used for the dispersion, $E_{p}=20-30 \mathrm{eV}$, provided the best compromise among surface sensitivity, high cross section for mode excitation, and $q_{\|}$resolution. As given by $\hbar q_{\|}=\hbar\left(k_{i} \sin \alpha_{i}-k_{s} \sin \alpha_{s}\right)$, the parallel momentum transfer $q_{\|}$depends on $E_{p}, E_{\text {loss }}, \alpha_{i}$, and $\alpha_{s}$ according to

$$
q_{\|}=\frac{\sqrt{2 m E_{p}}}{\hbar}\left(\sin \alpha_{i}-\sqrt{1-\frac{E_{\mathrm{loss}}}{E_{p}}} \sin \alpha_{s}\right),
$$

where $E_{\text {loss }}$ is the energy loss and $\alpha_{s}$ is the electron scattering angle [23]. Accordingly, the integration window in reciprocal space is [24]

$$
\Delta q_{\|} \approx \frac{\sqrt{2 m E_{p}}}{\hbar}\left(\cos \alpha_{i}+\sqrt{1-\frac{E_{\mathrm{loss}}}{E_{p}}} \cos \alpha_{s}\right) \gamma,
$$

where $\gamma$ is the angular acceptance of the apparatus [24] $\left( \pm 0.5^{\circ}\right.$ in our case). All measurements have been carried out at room temperature.

Figure 1(a) shows the HREELS spectra measured in specular geometry $\left(55^{\circ}\right.$ with respect to the sample normal) at various primary electron beam energies, in scattering conditions for which $q_{\|} \approx 0$. An intense loss feature is observed around $100 \mathrm{meV}$. The intensity of this feature depends strongly on the kinetic energy of the primary electrons. Its intensity increases with $E_{p}$, reaching a maximum at $E_{p}=25 \mathrm{eV}$. For higher values of the impingent energy, its intensity decreases and nearly vanishes at $E_{p}=40 \mathrm{eV}$. A deeper investigation of the nature of this mode may be achieved by probing the behavior of its cross section as a function of the scattering geometry. Measured loss spectra for various scattering geometries (with the incident angle ranging from $50^{\circ}$ to $80^{\circ}$ with respect the sample normal) are shown in Fig. 1(b). The intensity of this mode is enhanced in grazing scattering conditions, thus confirming its strict surface nature. We consider this excitation to be the surface plasmon of $\mathrm{Bi}_{2} \mathrm{Se}_{3}$. In view of the particular scattering conditions, the data set reported in Fig. 1 is devoid of spectral contributions from the Dirac plasmon, whose energy at $q_{\|} \approx 0$ is zero [25,26]. Moreover, it should be noted that the intensity of a Dirac plasmon

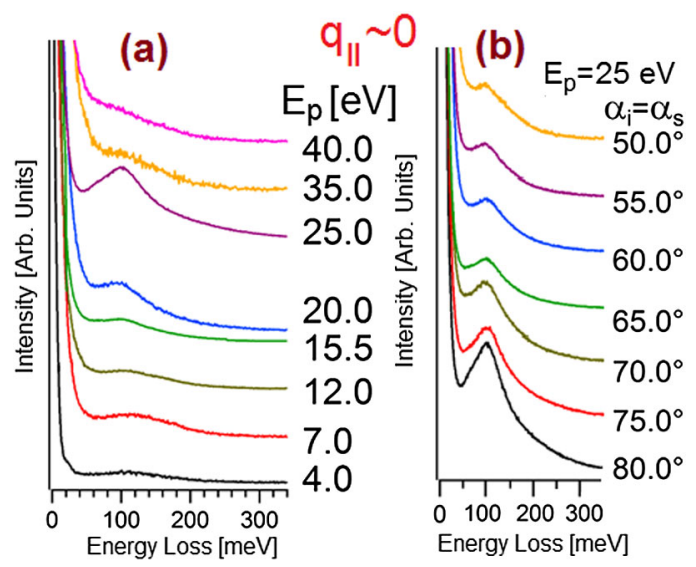

FIG. 1 (color online). (a) HREELS spectra measured for $\mathrm{Bi}_{2} \mathrm{Se}_{3}(0001)$ at various primary electron beam energies. The spectra were measured in specular geometry, with an incident angle of $55^{\circ}$ with respect to the sample normal. (b) HREELS spectra measured for various scattering geometries, for specular conditions (angles are measured with respect to the normal to the surface). The primary electron beam energy is $25 \mathrm{eV}$. 
vanishes at low momenta, as for any plasmonic excitation in a 2 DEG [26].

Momentum-resolved loss spectra can reveal the existence of the Dirac plasmon, which coexists with the surface plasmon. The HREELS spectra measured for various scattering angles, reported in Fig. 2(a), exhibit the presence of two clearly distinct plasmonic excitations. The dispersion relations of the modes were measured by varying the scattering angle and, consequently, the parallel momentum transfer. The energies of the loss peaks were obtained by subtracting a polynomial background from each spectrum. Finally, the resulting spectra were fitted by means of two Gaussian line shapes, as sketched in Fig. 2(b) for a selected case.

The measured dispersion of the aforementioned two plasmonic excitations is shown in Fig. 3. The surfaceplasmon energy $\omega_{\mathrm{sp}}$ of the first mode exhibits dispersion from $104 \mathrm{meV}$ in the long-wavelength limit up to $\sim 150 \mathrm{meV}$ at $q_{\|} \sim 0.28 \AA^{-1}$. For small momenta, a nearly flat dispersion of the surface-plasmon energy is found, while for higher momenta, starting from $q_{\|} \sim 0.04 \AA^{-1}$ (hereafter, we refer to this momentum value as to $q_{\|}^{c}$ ), the plasmon energy grows by $\Delta \omega_{\mathrm{sp}}\left(q_{\|}\right) \propto \sqrt{q_{\|}-q_{\|}^{c}}$ up to $q_{\|} \sim 0.2 \AA^{-1}$, where it becomes saturated. Note that at the momentum transfer $q_{\|}^{c}$, the second plasmonic mode also emerges and its peak becomes the dominant feature in the HREELS spectrum. It has near-linear dispersion from $\sim 140 \mathrm{meV}$ at $q_{\|}^{c}$ up to $\sim 200 \mathrm{meV}$ at $q_{\|} \sim 0.12 \AA^{-1}$.

As was found in previous experimental studies [27], the growth of $\mathrm{Bi}_{2} \mathrm{Se}_{3}$ intrinsically results in an $n$-type doping. We have characterized our samples by transport measurements (see the Supplemental Material [20] for details), finding that the 3D charge density of doping electrons for $\mathrm{Bi}_{2} \mathrm{Se}_{3}$ is $n_{3 \mathrm{D}} \sim 8.2 \times 10^{18} \mathrm{~cm}^{-3}$. In the long-wavelength limit, the bulk plasmon energy is defined as $\omega_{p}=$ $\sqrt{4 \pi n_{3 \mathrm{D}} / m^{*}}$ with the effective mass $m^{*}=0.15$, as evaluated in correspondence with the conduction band minimum
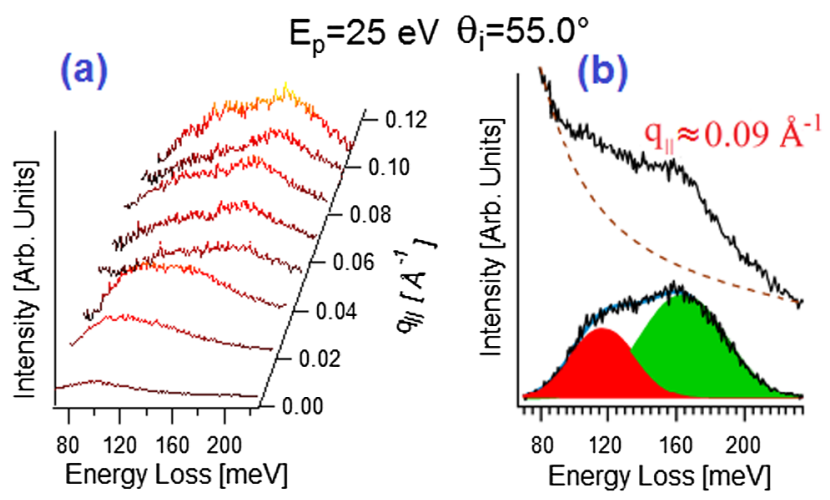

FIG. 2 (color online). (a) Momentum-resolved HREELS spectra. (b) After background subtraction, the raw HREELS spectrum recorded for $q_{\|}=0.09 \AA^{-1}$ (top spectrum) has been fitted with two Gaussian line shapes (bottom spectrum). The primary electron beam energy is $25 \mathrm{eV}$. at $\bar{\Gamma}$ [18]. This results in $\omega_{p}=270 \mathrm{meV}$. In general, bulk plasmons are determined in correspondence with the maxima of $\operatorname{Im}(-1 / \epsilon)$, which are shifted to higher energy with respect to maxima observed in optical absorption. The bulk plasmon in $\mathrm{Bi}_{2} \mathrm{Se}_{3}$ has been previously studied only by optical techniques [11,17], and a direct comparison with HREELS would not be straightforward. Nonetheless, an estimate, based on the Drude conductivity reported in Ref. [11], that the bulk plasmon energy is approximatively $\omega_{p}^{D} \sim 300 \mathrm{meV}$ supports satisfactory agreement.

In considering the system of 3D doping electrons having the characteristics indicated above, we constructed a freeelectron-like model to describe its dynamical response. This model incorporates information about the $\mathrm{Bi}_{2} \mathrm{Se}_{3}(0001)$ surface band structure in the vicinity of the Fermi level [as shown in the inset of Fig. 3(c)] while maintaining the relatively simple framework of the freeelectron gas. Our calculations based on this model employed a standard thick slab geometry (see, e.g., Ref. [28]) with experimental values of $m^{*}$ and $n_{3 \mathrm{D}}$, which accurately reproduce the experimentally observed value of $\omega_{\text {sp }}$ for low $q_{\|}$. The resulting surface loss function, whose peaks provide information about collective electronic excitations at the surface [29,30], was obtained analytically. As seen in Fig. 3(b), the calculated surface-plasmon energy dispersion of a purely 3D system is negative for low $q_{\|}$ values, as observed in other metallic systems [30]. At momenta exceeding a value of $\sim 0.05 \AA$, the dispersion becomes positive.

To take account of the electronic structure of the (0001) surface of bismuth selenide, our model incorporates a quasi-2D system formed by electrons in the surface state having an ideal Dirac cone eigenenergy spectrum. The dynamic, nonlocal screening properties of this system are described within the random phase approximation similarly to those of graphene (see, e.g., Refs. [31,32]) except that there is neither valley nor spin degeneracy in the case at hand. Additionally, we have taken into account the fact that this surface state is localized within the uppermost quintuple layer [33], in which the Coulomb interaction between surface-state electrons is screened by bulk electrons of $\mathrm{Bi}_{2} \mathrm{Se}_{3}$ (we employed the bulk dielectric constant of Ref. [34]). Figure 3(c) shows the Dirac-plasmon dispersion of the modeled 2D system.

The resulting calculated dispersion relation associated with peaks in the loss function of the composite system (bulk + Dirac fermions) is presented in Fig. 3(a). It is evident that the theoretical model reasonably represents the main experimental features measured in the HREELS spectrum. In the low wave number range $q_{\|}<q_{\|}^{c}$, the calculated loss function exhibits a single peak at energy around $105 \mathrm{meV}$, with initial dispersion that is comparable with experimental data. For momenta higher than $q_{\|}^{c}$, this peak acquires a slight positive dispersion. This slight deviation of experimental data from theoretical predictions 

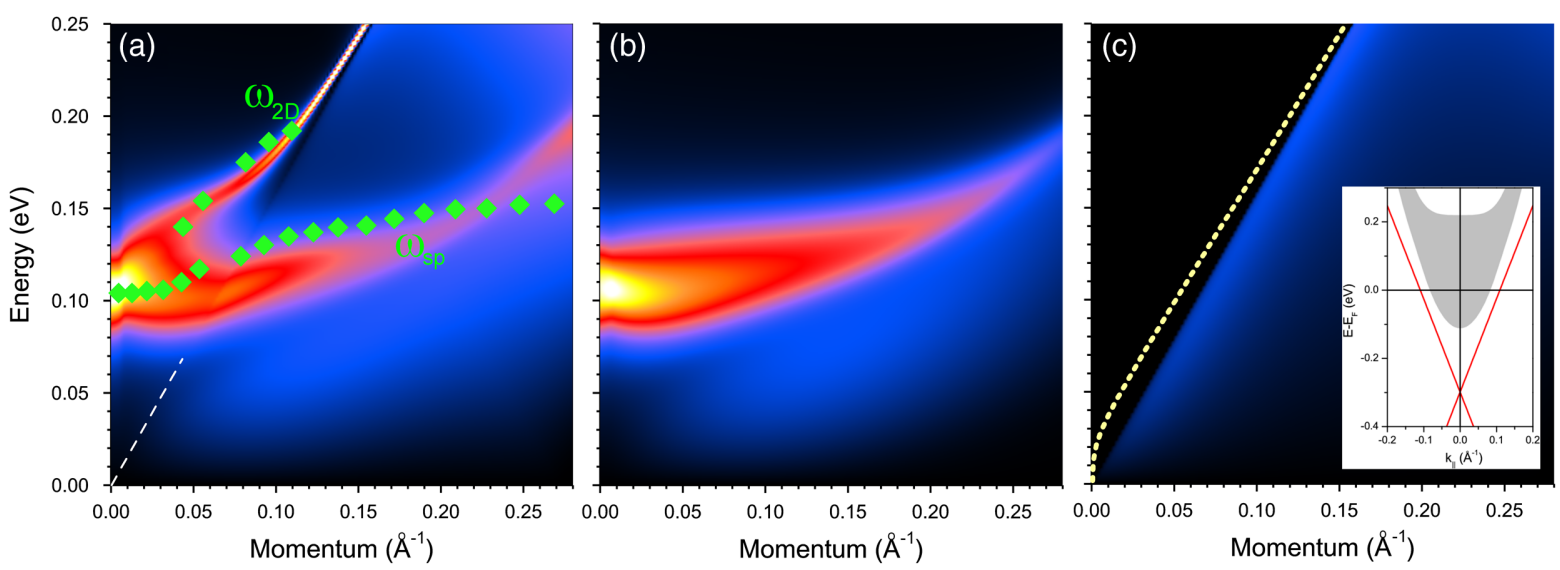

FIG. 3 (color online). (a) Coupled surface and Dirac plasmon dispersion relation measured on a $\mathrm{Bi}_{2} \mathrm{Se}_{3}(0001)$ surface. Green diamonds indicate peak positions when a two-peak fit is applied to the experimental spectrum. The intensity plot exhibits the surfaceloss function calculated for the two-component system (jellium and Dirac fermions). (b) Surface plasmon dispersion obtained for a single-component jellium model. (c) Dirac plasmon dispersion in a separate 2D Dirac electron system formed by surface-state electrons. Inset: Sketch of the modeled surface electronic structure of bismuth selenide.

occurring at larger momenta should likely be ascribed to transitions involving topologically protected surface states and three-dimensional states in the conduction band of $\mathrm{Bi}_{2} \mathrm{Se}_{3}$, which are not reproducible in our model.

In the calculated loss function, the second mode also emerges beyond $q_{\|}^{c}$. Its dispersion closely follows the experimental higher-energy peak, thus confirming that it corresponds to the Dirac plasmon. From a simple moderepulsion picture one may expect the presence of the Dirac plasmon at energies below $100 \mathrm{meV}$. The calculations reveal a tiny signature of such a mode, as illustrated by the dashed line in Fig. 3(a). However, its spectral weight is significantly reduced in comparison with that of the other modes. This may explain the absence of such a mode in the HREELS spectrum.

The chemical inertness of our $\mathrm{Bi}_{2} \mathrm{Se}_{3}$ samples [19] prevents the appearance of contamination-induced 2DEG states reported previously [35-37]. However, we would like to point out that no additional plasmonic mode would exist even in samples exhibiting 2DEG states because of their energy position at the bottom of the bulk conduction band. As a result, the maximum Fermi velocities in the bulk electron system and the conventional 2D electron system are very close, masking their distinction.

Finally, in regard to the prospect of TI-based infrared plasmonics, it should be remarked that present HREELS experiments demonstrate that the energy of the Dirac plasmon can be extended up to $\approx 200 \mathrm{meV}$, whereas in the previous studies [15] this energy region was restricted to the $0-15 \mathrm{meV}$ range.

In conclusion, we have investigated the plasmonic excitations of the (0001) surface of $\mathrm{Bi}_{2} \mathrm{Se}_{3}$. Our HREELS experiments indicate the presence of two plasmonic modes. The first one, having the plasmon energy of about $100 \mathrm{meV}$ in the long-wavelength limit, cannot be ascribed to a plasmon mode of a $2 \mathrm{D}$ electron system, but its surface nature clearly indicates that it is an ordinary surface plasmon related to the existence of 3D doping electrons in the sample. On the other hand, the second mode is a clear signature of the 2D Dirac-fermion system formed by surface-state electrons. Our theoretical results provide substantial support for the experimental coexistence and mutual interaction of the surface plasmon of the doped 3D electron system coupled with the Dirac plasmon of the 2D Dirac surface fermions. Our results provide insight concerning the dielectric properties of $\mathrm{Bi}_{2} \mathrm{Se}_{3}$ single crystals, the understanding of which is essential for the promising prospect of TI-based plasmonic devices, helping to bring to fruition a hitherto semiunexplored research field with high potential for technological applications.

We acknowledge funding from the University of Basque Country UPV/EHU (IT-756-13), the Departamento de Educación del Gobierno Vasco, the Tomsk State University Academic D. I. Mendeleev Fund Program (Grant No. 8.1.05.2015), the Spanish Ministry of Economy and Competitiveness MINECO (Grant No. FIS2013-48286-C2-1-P), Saint Petersburg State University (Project No. 11.50.202.2015), the Italian Ministry of Education, University, and Research (MIUR) through the program "FIRB"-Futuro in Ricerca 2010 RBFR10LULP "Fundamental research on Terahertz photonic devices." We also thank Fabio Vito for technical support.

*antonio.politano@fis.unical.it

[1] C. L. Kane and E. J. Mele, Phys. Rev. Lett. 95, 146802 (2005). 
[2] M. Z. Hasan and C. L. Kane, Rev. Mod. Phys. 82, 3045 (2010).

[3] X. L. Qi and S. C. Zhang, Rev. Mod. Phys. 83, 1057 (2011).

[4] K. Kuroda, M. Arita, K. Miyamoto, M. Ye, J. Jiang, A. Kimura, E. E. Krasovskii, E. V. Chulkov, H. Iwasawa, T. Okuda, K. Shimada, Y. Ueda, H. Namatame, and M. Taniguchi, Phys. Rev. Lett. 105, 076802 (2010).

[5] H. Lin, T. Das, Y. Okada, M. C. Boyer, W. D. Wise, M. Tomasik, B. Zhen, E. W. Hudson, W. Zhou, V. Madhavan, C. Y. Ren, H. Ikuta, and A. Bansil, Nano Lett. 13, 1915 (2013).

[6] J. Henk, A. Ernst, S. V. Eremeev, E. V. Chulkov, I. V. Maznichenko, and I. Mertig, Phys. Rev. Lett. 108, 206801 (2012).

[7] X. Yao, M. Tokman, and A. Belyanin, Phys. Rev. Lett. 112, 055501 (2014).

[8] F. H. L. Koppens, T. Mueller, Ph. Avouris, A. C. Ferrari, M. S. Vitiello, and M. Polini, Nat. Nanotechnol. 9, 780 (2014).

[9] R. H. Ritchie, Phys. Rev. 106, 874 (1957).

[10] D. Pines and P. Nozières, The Theory of Quantum Liquids (Benjamin, New York, 1966), Vol. I.

[11] K. W. Post, B. C. Chapler, L. He, X. Kou, K. L. Wang, and D. N. Basov, Phys. Rev. B 88, 075121 (2013).

[12] S. V. Dordevic, M.S. Wolf, N. Stojilovic, H. Lei, and C. Petrovic, J. Phys. Condens. Matter 25, 075501 (2013).

[13] S. Raghu, S. B. Chung, X.-L. Qi, and S.-C. Zhang, Phys. Rev. Lett. 104, 116401 (2010).

[14] T. Stauber, G. Gómez-Santos, and L. Brey, Phys. Rev. B 88, 205427 (2013).

[15] P. Di Pietro, M. Ortolani, O. Limaj, A. Di Gaspare, V. Giliberti, F. Giorgianni, M. Brahlek, N. Bansal, N. Koirala, S. Oh, P. Calvani, and S. Lupi, Nat. Nanotechnol. 8, 556 (2013).

[16] T. Kondo, Y. Nakashima, Y. Ota, Y. Ishida, W. Malaeb, K. Okazaki, S. Shin, M. Kriener, S. Sasaki, K. Segawa, and Y. Ando, Phys. Rev. Lett. 110, 217601 (2013).

[17] P. Di Pietro, F. M. Vitucci, D. Nicoletti, L. Baldassarre, P. Calvani, R. Cava, Y. S. Hor, U. Schade, and S. Lupi, Phys. Rev. B 86, 045439 (2012).

[18] N. P. Butch, K. Kirshenbaum, P. Syers, A. B. Sushkov, G. S. Jenkins, H. D. Drew, and J. Paglione, Phys. Rev. B 81, 241301 (2010).

[19] A. Politano, M. Caputo, S. Nappini, F. Bondino, E. Magnano, Z.S. Aliev, M. B. Babanly, A. Goldoni,
G. Chiarello, and E. V. Chulkov, J. Phys. Chem. C 118, 21517 (2014).

[20] See Supplemental Material at http://link.aps.org/ supplemental/10.1103/PhysRevLett.115.216802, which includes Refs. [21,22], for details of the measurements of the 3D charge density of doping electrons.

[21] V. A. Golyashov, K. A. Kokh, S. V. Makarenko, K. N. Romanyuk, I. P. Prosvirin, A. V. Kalinkin, O. E. Tereshchenko, A. S. Kozhukhov, D. V. Sheglov, S. V. Eremeev, S. D. Borisova, and E. V. Chulkov, J. Appl. Phys. 112, 113702 (2012).

[22] D. Kong, J. J. Cha, K. Lai, H. Peng, J. G. Analytis, S. Meister, Y. Chen, H. J. Zhang, I. R. Fisher, Z. X. Shen, and Y. Cui, ACS Nano 5, 4698 (2011).

[23] A. Politano, G. Chiarello, G. Benedek, E. V. Chulkov, and P. M. Echenique, Surf. Sci. Rep. 68, 305 (2013).

[24] M. Rocca, Surf. Sci. Rep. 22, 1 (1995).

[25] F. Stern, Phys. Rev. Lett. 18, 546 (1967).

[26] T. Nagao, T. Hildebrandt, M. Henzler, and S. Hasegawa, Phys. Rev. Lett. 86, 5747 (2001).

[27] Z. Wang, T. Lin, P. Wei, X. Liu, R. Dumas, K. Liu, and J. Shi, Appl. Phys. Lett. 97, 042112 (2010).

[28] V. M. Silkin, J. M. Pitarke, E. V. Chulkov, and P. M. Echenique, Phys. Rev. B 72, 115435 (2005).

[29] B. N. J. Persson and E. Zaremba, Phys. Rev. B 31, 1863 (1985).

[30] A. Liebsch, Electronic Excitations at Metal Surfaces (Plenum Press, New York, 1997).

[31] B. Wunsch, T. Stauber, F. Sols, and F. Guinea, New J. Phys. 8, 318 (2006).

[32] E. H. Hwang and S. Das Sarma, Phys. Rev. B 75, 205418 (2007).

[33] S. V. Eremeev, Yu. M. Koroteev, and E. V. Chulkov, JETP Lett. 91, 387 (2010).

[34] I. A. Nechaev, R. C. Hatch, M. Bianchi, D. Guan, C. Friedrich, I. Aguilera, J. L. Mi, B. B. Iversen, S. Blügel, Ph. Hofmann, and E. V. Chulkov, Phys. Rev. B 87, 121111 (R) (2013).

[35] M. Bianchi, D. Guan, S. Bao, J. Mi, B. B. Iversen, P. D. C. King, and P. Hofmann, Nat. Commun. 1, 128 (2010).

[36] C. E. ViolBarbosa, C. Shekhar, B. Yan, S. Ouardi, E. Ikenaga, G. H. Fecher, and C. Felser, Phys. Rev. B 88, 195128 (2013).

[37] J. Duan, N. Tang, X. He, Y. Yan, S. Zhang, X. Qin, X. Wang, X. Yang, F. Xu, Y. Chen, W. Ge, and B. Shen, Sci. Rep. 4, 4889 (2014). 